\title{
Comparação dos Resultados entre os Fenômenos de No-Reflow e Slow-Flow Coronariano em Pacientes sem IAMSSST
}

\author{
Comparison of the Outcomes between Coronary No-Reflow and Slow-Flow Phenomenon in Non-STEMI Patients
}

Mustafa Ahmet Huyut ${ }^{10}$

Yeni Yuzyil University, Faculty of Medicine, Department of Cardiology, ${ }^{1}$ Istambul - Turquia

\section{Resumo}

Fundamento: Os fenômenos de slow-flow (CSFP) e no-reflow coronariano (CNP) estão associados a um risco aumentado de eventos cardiovasculares adversos maiores (ECAM).

Objetivos: Este estudo teve como objetivo avaliar e comparar os resultados do seguimento clínico de um ano entre pacientes com CNP e CSFP submetidos a intervenções coronárias percutâneas (ICP) em infarto agudo do miocárdio sem supradesnivelamento do segmento ST (IAMSSST).

Métodos: Este estudo incluiu um total de 858 pacientes com diagnóstico de IAMSSST e submetidos a ICP nas 24 horas desde o início dos sintomas. Os pacientes foram divididos em dois grupos, o grupo CSFP $(n=221)$ e o grupo CNP ( $n=25)$, considerando as características angiográficas do fluxo da trombólise no infarto do miocárdio (TIMI) e na artéria relacionada ao infarto. Os pacientes tiveram um seguimento de um ano. Um valor de $p<0,05$ foi considerado significativo.

Resultados: O CNP foi observado em 2,91\% e o CSFP em 25,75\% dos pacientes. Os desfechos clínicos analisaram que a incidência de acidente vascular cerebral (AVC) foi significativamente maior no grupo CNP do que no grupo CSFP (6 (24\%) vs. $6(2,70 \%)$, p < 0,001$)$ e a de ECAM foi significativamente maior no grupo CNP do que no grupo CSFP (11 (44\%) vs. $51(23,10 \%), p=0,022)$. A análise de regressão logística condicional forward demonstrou que o índice de massa corporal $(I M C)(O R=1,11, I C 95 \%: 1,00-1,24, p=0,038)$ e frequência cardíaca $(F C)$ basal $(O R=0,923$, IC 95\%: 0,88$0,96, p<0,001)$ foram os preditores independentes de CNP no IAMSSST.

Conclusões: Pacientes com CNP têm piores resultados clínicos e um maior risco de AVC em comparação com pacientes com CSFP no IAMSSST. (Arq Bras Cardiol. 2021; 116(5):856-864)

Palavras-chave: Infarto do Miocárdio; Síndrome Coronariana Aguda; Fenômeno de Não Refluxo; Intervenção Coronária Percutânea/complicações; Fatores de Risco; Angiografia Coronária; Acidente Vascular Cerebral.

\section{Abstract}

Background: Coronary slow-flow phenomenon (CSFP) and coronary no-reflow phenomenon (CNP) are associated with an increased risk of major cardiovascular adverse events (MACE).

Objectives: This study aimed to evaluate and compare the one-year clinical follow-up outcomes between patients with CNP and CSFP undergoing percutaneous coronary interventions (PCI) in non-ST elevation myocardial infarction (NSTEMI).

Methods: This study included a total of 858 patients who were diagnosed with NSTEMI and underwent PCI within $24 \mathrm{~h}$ of symptom onset. The patients were divided into two groups, the CSFP group $(n=221)$ and the CNP group $(n=25)$, regarding the angiographic characteristics of thrombolysis in myocardial infarction (TIMI) flow of the infarct-related artery. Patients were followed for one-year. A p-value of $<0.05$ was considered significant.

Results: CNP was observed in 2.91\%, and CSFP was observed in 25.75\% of the patients. Clinical endpoints analyzed that stroke was significantly higher in the CNP group than in the CSFP group (6 $(24 \%)$ vs. $6(2.70 \%), p<0.001)$ and MACE was significantly higher in the CNP group than in the CSFP group (11 (44\%) vs. $51(23.10 \%), p=0.022)$. Forward conditional logistic regression analysis demonstrated that body mass index $(B M I)(O R=1.11,95 \% C l: 1.00-1.24, p=0.038)$ and baseline heart rate $(H R)(O R=0.923,95 \% C l: 0.88-0.96, p<0.001)$ were the independent predictors of CNP in NSTEMI.

Correspondência: Mustafa Ahmet Huyut •

Yeni Yuzyil University, Faculty of Medicine, Department of Cardiology, Merkez Mah. Cukurcesme Caddesi No:51 Gaziosmanpasa Istanbul - Turquia E-mail: ahuyut@yahoo.com

Artigo recebido em 24/12/2019, revisado em 19/02/2020, aceito em 08/04/2020

DOI: https://doi.org/10.36660/abc.20190905 
Conclusion: CNP patients have worse clinical outcomes and a higher risk of stroke compared with CSFP patients in NSTEMI. (Arq Bras Cardiol. 2021; 116(5):856-864)

Keywords: bMyocardial Infarction; No-Reflow Phenomenon; Percutaneous Coronary Interventon; Acute Coronary Syndrome/complications; Risk Factors; Coronary Angiography; Stroke.

Full texts in English - http://www.arquivosonline.com.br

\section{Introdução}

As síndromes coronárias agudas continuam sendo uma das principais causas de mortalidade e morbidade nos países industrializados e estão se tornando um problema cada vez mais importante nos países em desenvolvimento, apesar das melhoras em seu manejo e sua prevenção. ${ }^{1}$ Entre as síndromes coronárias agudas, os pacientes com infarto agudo do miocárdio sem supradesnivelamento do segmento ST (IAMSSST) apresentam os piores desfechos de longo prazo. ${ }^{2}$ Poucos estudos, entretanto, relataram os desfechos no IAMSSST, e esses relatos não esclareceram a diferença entre as características dos subgrupos fenômeno de slow-flow (CSFP, do inglês coronary slow-flow phenomenon) e fenômeno de no-reflow coronariano (CNP, do inglês coronary no-reflow phenomenon) na prática clínica, tanto no hospital quanto no seguimento de longo prazo, dentro de uma perspectiva do 'mundo real' ${ }^{3,4} \mathrm{Na}$ ausência de doença arterial coronária obstrutiva, o fluxo coronário TIMI-II e a opacificação coronária tardia são definidos como CSFP. ${ }^{5}$ Além disso, o fluxo TIMI 0-I sem dissecção, obstrução mecânica, estenose residual significativa, espasmo ou trombo da artéria coronária são definidos como CNP angiográfico. ${ }^{6}$ Os mecanismos subjacentes no CNP e CSFP são inflamação, microembolização aterotrombótica, ativação de neutrófilos e plaquetária, que desencadeiam a liberação de radicais livres de oxigênio, enzimas proteolíticas e mediadores próinflamatórios, que podem causar dano tecidual e endotelial especialmente em miócitos com lesões graves. ${ }^{5,6}$

Além disso, não está claro sob que circunstâncias as diferenças nas características clínicas e nos desfechos persistem em pacientes com IAMSSST. Também não há evidências na literatura sobre como o fenômeno de slow-flow pode afetar os resultados no IAMSSST. Além disso, a comparação dos resultados entre CSFP e CNP em pacientes com IAMSSST não foi abordada na literatura. Nossa hipótese é que os piores desfechos clínicos no IAMSSST estão fortemente relacionados ao não-fluxo TIMI III nas artérias coronárias e especialmente no subconjunto do grupo CNP. No presente estudo, nosso objetivo foi investigar as características clínicas e comparar os principais desfechos cardiovasculares entre os grupos CSFP e CNP em pacientes com IAMSSST que foram seguidos por 12 meses.

\section{Métodos}

Para este estudo de centro único, conduzido prospectivamente, um total de 858 pacientes com idade entre 18 e 90 anos foi incluído entre junho de 2016 e junho de 2018 no Bezmialem University Hospital, que foram diagnosticados com IAMSSST e submetidos a ICP precoce nas 24 horas desde o início dos sintomas (Figura1). Pacientes com fluxo
TIMI III, cirurgia de revascularização do miocárdio (CABG), choque cardiogênico, edema pulmonar, sinais de disfunção ventricular esquerda aguda, trombose de stent, submetidos a aspiração de trombo no evento índice, com doença infecciosa ou neoplásica aguda ou crônica, doença renal moderadagrave e doença hepática crônica foram excluídos deste estudo ( $n=602$ ). De acordo com os resultados finais das características angiográficas do fluxo TIMI da artéria culpada tratada, um total de 25 pacientes com CNP comprovado por angiografia foram inscritos no grupo CNP, enquanto 221 pacientes com comprovação angiográfica de CSFP foram inscritos no grupo CSFP. Todos os pacientes receberam um total de $300 \mathrm{mg}$ de ácido acetilsalicílico e uma dose de ataque $(600 \mathrm{mg})$ de clopidogrel e heparina NF $(100 \mathrm{mg} / \mathrm{kg})$ durante a ICP. As informações de seguimento foram obtidas de registros hospitalares e após 1, 3, 6 e 12 meses durante as consultas feitas pelo paciente com o mesmo investigador. Os endpoints deste estudo foram obtidos a partir de prontuários hospitalares e atestados de óbito ou contato telefônico com parentes dos pacientes. Os eventos cardiovasculares adversos maiores (ECAM) foram definidos como mortalidade por todas as causas, morte cardiovascular, acidente vascular cerebral e reinfarto do miocárdio. Todos os participantes deram consentimento informado por escrito antes da participação no estudo e o estudo foi aprovado pelo comitê de ética local (Número: 7/70-04/17). Além disso, o estudo foi conduzido de acordo com os princípios da Declaração de Helsinque.

\section{Avaliação Bioquímica}

Amostras de sangue venoso foram coletadas da veia antecubital imediatamente após a hospitalização antes da ICP. Um eletrocardiograma de 12 derivações foi obtido no momento da hospitalização no pronto-socorro e a frequência cardíaca (FC) foi registrada. A taxa de filtração glomerular estimada (TFGe) de cada paciente foi calculada utilizando-se a equação Chronic Kidney Disease Epidemiology Collaboration. O IMC foi calculado através da fórmula: peso $(\mathrm{kg}) /$ altura ${ }^{2}$ $\left(\mathrm{m}^{2}\right)$. A avaliação bioquímica de rotina do sangue, parâmetros lipídicos e níveis de troponina I cardíaca foram medidos em um autoanalisador padrão. O hemograma foi realizado com um autoanalisador Sysmex K-1000 (Block Scientific, Bohemia, NY, EUA). As amostras foram centrifugadas a $3000 \mathrm{rpm}$ durante $10 \mathrm{~min}$, e o sobrenadante e o soro foram separados das amostras, e em seguida, foram congelados a $-80^{\circ} \mathrm{C}$ até análise posterior. A medida dos níveis de creatinina sérica foi repetida após 72 horas, depois da administração do meio de contraste (MC). A nefropatia induzida por contraste foi definida como um aumento absoluto de $0,5 \mathrm{mg} / \mathrm{dL}$ no nível de creatinina sérica acima da linha basal ou $\geq 25 \%$ de aumento relativo no nível de creatinina sérica basal nas 72 horas de exposição ao MC. 


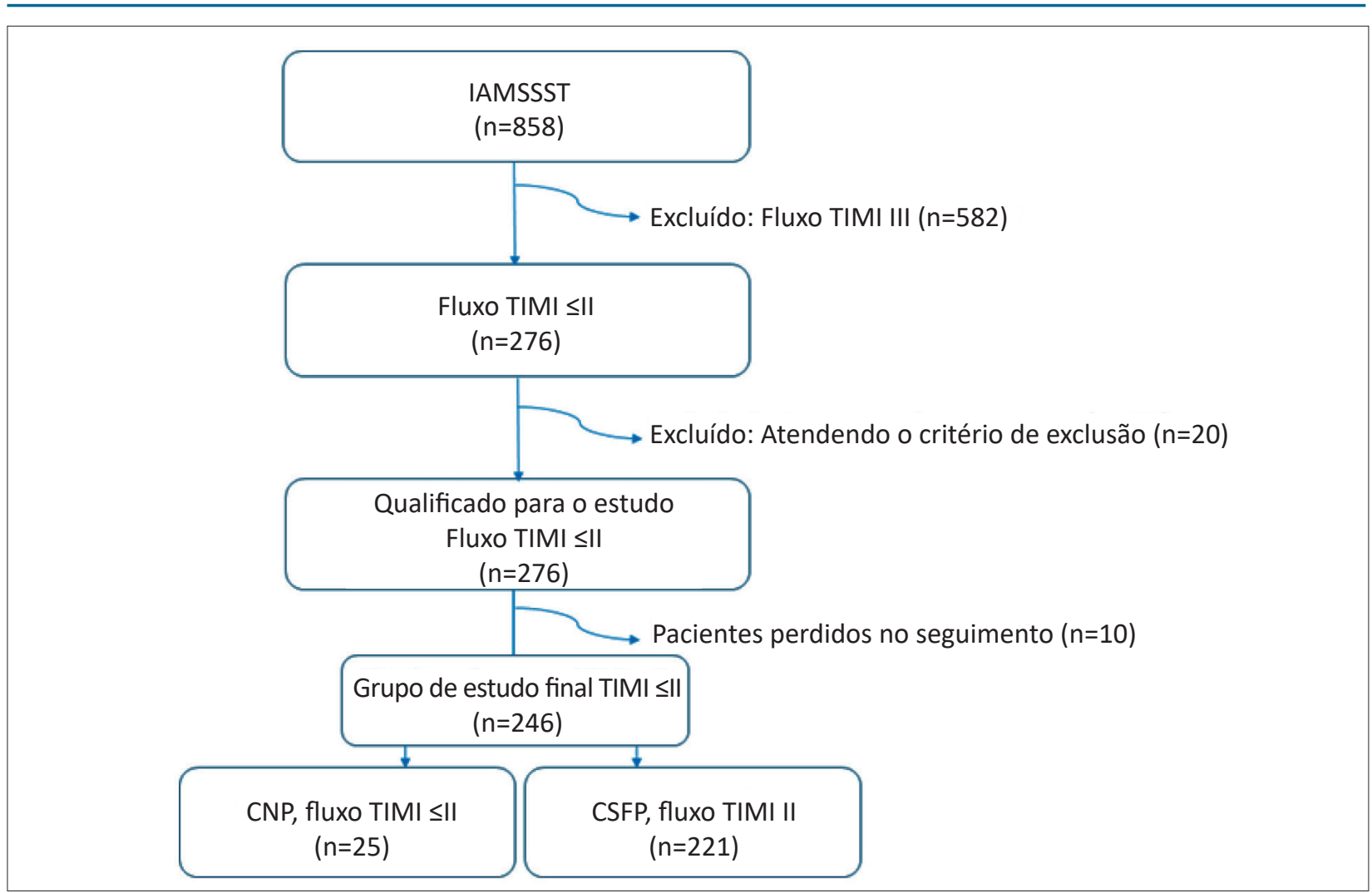

Figura 1 - Diagrama mostrando a seleção dos grupos de estudo. IAMSSST: infarto agudo do miocárdio sem supradesnivelamento do segmento ST; TIMI: trombólise no infarto do miocárdio; CNP: fenômeno no-reflow coronariano; CSFP: fenômeno slow-flow coronariano.

Diagnóstico de infarto do miocárdio sem elevação do segmento ST

O diagnóstico de IAMSSST foi feito na presença das seguintes características com base nas definições das diretrizes de prática clínica. ${ }^{7}$ Os pacientes com IAMSSST apresentaram dor ou desconforto torácico típico em repouso ou com esforço mínimo por pelo menos 10 minutos e o ECG inicial mostrou um ECG normal ou alterações isquêmicas, como infradesnivelamento do segmento ST ou inversão da onda T com nível elevado de troponina I cardíaca com pelo menos um valor acima do limite superior de referência do percentil 99.

\section{Fatores de Risco Cardiovascular}

Após exames detalhados, o histórico médico de cada paciente foi coletado pelo mesmo investigador. Fatores de risco foram identificados para doença arterial coronariana (DAC), fatores de risco cardiovascular, incluindo idade, sexo, diabetes mellitus (DM), hipertensão (HT), hiperlipidemia (HPL) e tabagismo. Pacientes recebendo terapia anti-hipertensiva anterior ou com níveis de pressão arterial $\geq 140 / 90 \mathrm{mmHg}$ em pelo menos duas medidas, foram considerados hipertensos. ${ }^{8}$ Pacientes previamente tratados com antidiabético oral e/ou terapia com insulina ou cuja glicemia de jejum era $\geq 125$ $\mathrm{mg} / \mathrm{dL}$ em pelo menos duas medidas foram considerados diabéticos. ${ }^{9}$ A presença de HPL foi considerada quando foi obtida uma medida de colesterol total $>200 \mathrm{mg} / \mathrm{dL}$ ou de lipoproteína de baixa densidade-colesterol (LDL-C)>100 $\mathrm{mg} / \mathrm{dL}$, ou quando o paciente utilizava medicamento hipolipemiante, de acordo com a diretriz "Adult Treatment Panel III". ${ }^{10}$ Pacientes que ainda utilizavam produtos do tabaco na admissão ao serviço de emergência e aqueles que tinham parado de fumar no último mês foram considerados fumantes.

\section{Ecocardiografia Transtorácica}

Antes da alta, cada paciente foi submetido a um exame ecocardiográfico transtorácico utilizando um transdutor de 3,5 MHz (Vivid 7 GE Medical System, Horten, Noruega). Os exames e avaliações foram realizados de acordo com as recomendações das diretrizes da American Echocardiography Unit. O método de Simon foi utilizado para calcular a fração de ejeção do ventrículo esquerdo (FEVE). ${ }^{11}$

\section{Angiografia Coronária}

Os procedimentos da angiografia coronária foram realizados por via femoral utilizando o sistema de angiografia Philips (Optimus 200 DCA e Integris Allura 9, Philips Medical Systems, Eindhoven, Países Baixos). A angiografia coronária e a ICP foram realizadas com meio de contraste não iônico, isoosmolar (iodixanol, Visipaque 320mg/100mL, GE Healthcare, Cork, Irlanda) de acordo com as práticas clínicas padrão. A ICP da artéria relacionada ao infarto foi realizada. As imagens angiográficas foram obtidas a uma taxa de pelo menos 80 
quadros e gravadas a uma taxa de 25 quadros por segundo. Pelo menos dois cardiologistas especialistas examinaram a anatomia coronária e o grau de fluxo TIMI offline. O fluxo TIMI das coronárias foi determinado pelo número quantitativo da contagem de quadros como descrito por Gibson et al. ${ }^{12}$ Fluxos TIMI de 0-I sem dissecção, obstrução mecânica, estenose residual significativa, espasmo ou trombo da artéria coronária foram definidos como CNP angiográfico. Na ausência de doença arterial coronária obstrutiva, o fluxo coronário TIMI-II e a opacificação coronária tardia foram definidos como CSFP. Os pacientes com CNP receberam tratamento com inibidores da glicoproteína IIb/IIla intracoronários (IC) (inib. da Gp-IIb/ IIla ) ou adenosina IC ou epinefrina IC. Após o procedimento, todos os pacientes receberam hidratação venosa (IV) com solução salina isotônica $(1 \mathrm{~mL} / \mathrm{kg} / \mathrm{h})$ por pelo menos 12 horas.

\section{Análise Estatística}

A análise dos dados foi realizada com o pacote de software estatístico SPSS versão 22.0 (SPSS Inc., Chicago, IL, EUA). A distribuição normal de uma variável contínua foi avaliada pelo teste de Kolmogorov-Smirnov. O teste $t$ para amostras independentes ou o teste $U$ de MannWhitney foram utilizados para comparar variáveis contínuas dependendo se os pressupostos estatísticos fossem cumpridos ou não. As variáveis contínuas foram expressas em média e desvio padrão se distribuídas normalmente, ou medianas e percentis 25 e 75 caso não satisfizessem a suposição de normalidade. As variáveis categóricas foram expressas em número (porcentagem). O teste de qui-quadrado foi utilizado para comparar variáveis categóricas. A correlação entre as variáveis foi realizada através da análise do teste de log-rank de Spearman. O método de Kaplan-Meier foi utilizado para estimar as taxas de sobrevida livre de eventos. A análise da curva característica de operação do receptor (ROC, do inglês Receiver Operating Characteristic) foi realizada para determinar o valor preditivo do IMC e da FC para o CNP. Foi realizada a análise de regressão logística univariada, e as variáveis que se mostraram estatisticamente significativas $(p<0,1)$ foram avaliadas com a análise de regressão logística multivariada. O odds ratio e o intervalo de confiança de 95\% de cada variável independente foram calculados. Um valor de $\mathrm{P}$ bicaudal $<0,05$ foi considerado significativo.

\section{Resultados}

Neste estudo, incluímos um total de 858 pacientes com IAMSSST e, por fim, concluímos o presente estudo com 246 pacientes (171 homens; média de idade: 61,69 \pm 12,60 anos). Em pacientes com IAMSSST, o CNP foi observado em 2,91\% ( $n=25)$ e o CSFP foi observado em $25,75 \%$ ( $=221$ ). Para a população final do estudo, o grupo CNP teve $25(10,16 \%)$ pacientes e o grupo CSFP, 221 (89,84\%) pacientes. Os achados demográficos são descritos na Tabela 1. Além disso, a classe NYHA, a frequência cardíaca, o tempo de internação hospitalar, o escore de Mehran e a TFGe foram significativamente associados ao EuroSCORE-II $(p<0,05)$ (Tabela 2). Os achados do seguimento clínico foram descritos na Tabela 3. Não identificamos nenhum acidente vascular cerebral (AVC) hemorrágico durante o seguimento.
As estimativas de Kaplan-Meier para as taxas de AVC e ECAM são descritas na Figura 2A e Figura 2B. A análise de regressão logística condicional forward demonstrou que o IMC e a FC foram os preditores independentes de CNP (Tabela 4).

$\mathrm{Na}$ análise $\mathrm{ROC}$, um IMC $>28,38 \mathrm{~kg} / \mathrm{m}^{2}$ previu a presença de CNP com $80 \%$ de sensibilidade e 54\% de especificidade. A área sob a curva foi de 0,649 (IC 95\%: 0,548-0,750,p $=0,015$ ) (Figura 3A). Além disso, a FC $<66,5 \mathrm{bpm}$ previu a presença de CNP com $86 \%$ de sensibilidade e $60 \%$ de especificidade. A área sob a curva foi de 0,741 (IC 95\%: 0,88-0,96, p <0,001) (Figura 3B).

\section{Discussão}

O principal achado desta pesquisa foi que os dois determinantes do CNP em pacientes com IAMSSST foram os níveis aumentados de IMC e FC mais baixa. Além disso, em pacientes com IAMSSST, o CNP foi significativamente associado a desfechos ruins. Mostramos que os valores de IMC $>28,38 \mathrm{~kg} / \mathrm{m}^{2}$ sugerem a presença de CNP no IAMSSST. Além disso, a FC $<66,5$ bpm sugere a presença de CNP no IAMSSST. Que seja de nosso conhecimento, este é o primeiro relato na literatura que demonstra a relação entre o IMC e FC mais baixa em pacientes com CNP com IAMSSST. Em nosso estudo, os resultados do seguimento clínico de um ano mostraram que as incidências de AVC e ECAM foram significativamente maiores no grupo CNP. Neste estudo, mostramos que o CNP piorou os desfechos dos pacientes com IAMSSST.

CSFP e CNP não são achados frequentes, com uma incidência de aproximadamente $1 \%$ em pacientes submetidos à angiografia coronariana; entretanto, em relação aos dados publicados, as frequências estimadas de CNP e CSFP variam de $1 \%$ a $60 \%$ na síndrome coronariana aguda. ${ }^{13,14}$ Neste estudo, o CNP foi observado em 2,91\% e o CSFP em 25,75\% da população estudada. CSFP e CNP estão associados a desfechos clínicos ruins em curto e longo prazo. ${ }^{15} \mathrm{Em}$ particular, o CNP é um preditor significativo de desfechos cardíacos ruins no IAMSSST. ${ }^{13,16}$ Consistente com os dados publicados, encontramos os piores desfechos no grupo CNP. Em nosso estudo, os achados do seguimento clínico de um ano demonstraram que os desfechos de ECAM e AVC foram significativamente maiores no grupo CNP. No grupo CNP, a probabilidade de AVC foi 8,88 vezes maior do que no grupo CSFP.

Além disso, no grupo CNP, observamos uma probabilidade de ECAM 1,90 vezes maior do que no grupo PFC. Meta-análises anteriores, incluindo estudos retrospectivos e prospectivos, encontraram uma associação positiva entre troponina cardíaca e eventos adversos no IAMSSST. ${ }^{17}$ Neste estudo, consistente com a literatura, encontramos um nível de pico de troponina-I significativamente mais alto no grupo CNP. Enquanto isso, o acidente vascular cerebral foi associado à carga de um trombo. De acordo com nossa pesquisa, o mecanismo relacionado que causa esse evento adverso é a ativação contínua do trombo após o evento índice, e consideramos que essa pode ser a principal razão para o aumento do risco de acidente vascular cerebral. Embora todos os pacientes com IAMSSST tenham sido tratados regularmente com drogas antitrombóticas, o 


\begin{tabular}{|c|c|c|c|}
\hline Variável, n (\%) & CNP, n=25 (10.16) & CSFP, n=221 (89.84) & p-valor \\
\hline Idade, a & $66,28 \pm 14,14$ & $61,17 \pm 12,34$ & 0,057 \\
\hline Sexo feminino, $\mathrm{n}(\%)$ & $12(48)$ & $63(28,50)$ & 0,045 \\
\hline $\mathrm{IMC}, \mathrm{kg} / \mathrm{m}^{2}$ & $30,51 \pm 3,99$ & $28,34 \pm 4,55$ & 0,015 \\
\hline HT, n (\%) & $19(76)$ & $129(58,40)$ & 0,088 \\
\hline $\mathrm{DM}, \mathrm{n}(\%)$ & $10(40)$ & $70(31,70)$ & 0,400 \\
\hline HL, n (\%) & $9(36)$ & $95(43)$ & 0,503 \\
\hline Fumante, n (\%) & $15(60)$ & $132(59,70)$ & 0,979 \\
\hline Histórico familiar, n (\%) & $8(32)$ & $73(33)$ & 0,917 \\
\hline DAP, n (\%) & $5(20)$ & $13(5,90)$ & 0,010 \\
\hline DPOC, n (\%) & $5(20)$ & $31(14)$ & 0,423 \\
\hline FEVE, $\%$ & $50 \pm 7,40$ & $52,29 \pm 7,19$ & 0,126 \\
\hline Glicose, mg/dL & $115(90,50-174)$ & $106(96-146)$ & 0,719 \\
\hline Ácido úrico, mg/dL & $5,60(4,55-7,25)$ & $5,80(4,20-6,90)$ & 0,303 \\
\hline Creatinina, $\mathrm{mg} / \mathrm{dL}$ & $0,86(0,77-1,23)$ & $0,87(0,76-1,05)$ & 0,175 \\
\hline TFGe, $\mathrm{mL} / \mathrm{min}$ por $1,73 \mathrm{~m}^{2}$ & $70,90 \pm 25,95$ & $82,86 \pm 20,80$ & 0,021 \\
\hline Triglicérides, mg/dL & $153(125-195)$ & $147(110,5-180)$ & 0,353 \\
\hline $\mathrm{LDL}, \mathrm{mg} / \mathrm{dL}$ & $135(114-171)$ & $125(98-149)$ & 0,051 \\
\hline HTC, $\%$ & $40,60(35,80-42)$ & $41(37,10-43,15)$ & 0,344 \\
\hline Plaquetas, $10^{3} / \mathrm{uL}$ & $220(185-266)$ & $225(190-276,50)$ & 0,428 \\
\hline Pico de troponina-l, pg/mL & $814(156-5693,50)$ & $146(116-2113)$ & 0,037 \\
\hline PCR-us, mg/dL & $0,10(0,01-0,57)$ & $0,18(0,04-0,50)$ & 0,836 \\
\hline Frequência cardíaca, bpm & $69,60 \pm 19,86$ & $78,81 \pm 13,46$ & $<0,001$ \\
\hline Tempo de hospitalização, d. & $3,40 \pm 0,95$ & $3,00 \pm 0,88$ & 0,015 \\
\hline Escore de Mehran & $7,56 \pm 6,20$ & $5,24 \pm 4,91$ & 0,017 \\
\hline Desenvolvimento NIC, n (\%) & $4(16)$ & $19(8,60)$ & 0,228 \\
\hline Classe NYHA & $2,48 \pm 0,50$ & $2,04 \pm 0,40$ & $<0,001$ \\
\hline EuroSCORE II,\% & $3,96 \pm 3,95$ & $2,14 \pm 2,32$ & $<0,001$ \\
\hline \multicolumn{4}{|l|}{ Medicamentos, n (\%) } \\
\hline Inibidores de ECA & $17(68)$ & $110(49,80)$ & 0,084 \\
\hline BRA & $7(28)$ & $75(33,90)$ & 0,551 \\
\hline Betabloqueador & $24(96)$ & $212(95,90)$ & 0,986 \\
\hline $\mathrm{BCC}$ & $9(36)$ & $52(23,50)$ & 0,171 \\
\hline Estatina & $25(100)$ & $194(87,80)$ & 0,064 \\
\hline Nitrato & $11(44)$ & $73(33)$ & 0,273 \\
\hline DAOs & $10(40)$ & $68(30,80)$ & 0,347 \\
\hline Diuréticos & $13(52)$ & $71(32,10)$ & 0,047 \\
\hline Gp-Ilb / IIla inh. IC & $25(100)$ & $8(3,61)$ & $<0,001$ \\
\hline Adenosina IC & $25(100)$ & $1(0,45)$ & $<0,001$ \\
\hline Epinefrina IC & $25(100)$ & $1(0,45)$ & $<0,001$ \\
\hline
\end{tabular}

Os valores são expressos em média $\pm D P$ ou números e porcentagens ou mediana e percentis 25-75. Valor de $p$ para dados categóricos do Quiquadrado. Valor de $p$ para o teste $t$ de amostras independentes ou o teste $U$ de Mann-Whitney foi utilizado para comparar variáveis contínuas. CNP: fenômeno no-reflow coronariano; CSFP: fenômeno slow-flow coronariano; a: anos; IMC: Índice de Massa Corporal; HT: hipertensão; DM: diabetes mellitus tipo 2; HL: hiperlipidemia; DAP: doença arterial periférica; DPOC: doença pulmonar obstrutiva crônica; FEVE: fração de ejeção do ventrículo esquerdo; TFGe: taxa de filtração glomerular estimada; LDL: lipoproteína de baixa densidade; HTC: hematócrito; PCR-us: proteína C reativa de alta sensibilidade; bpm: batimentos por minuto; d: dias; NIC: Nefropatia induzida por contraste; NYHA: Classificação Funcional da New York Heart Association; EuroSCORE: European System for Cardiac Operative Risk Evaluation; Inibidores ECA: inibidores da enzima de conversão da angiotensina; BRA: bloqueadores do receptor da angiotensina; B bloqueador: betabloqueador; BCC. bloqueadores dos canais de cálcio; DAOs: drogas anti-hiperglicêmicas orais; IC: intracoronário; Inibidores Gp-Ilb / Illa: inibidores da glicoproteína-IIb/IIla. 
Artigo Original

\begin{tabular}{lcc}
\hline Tabela 2 - Características basais significativamente associadas ao EuroSCORE II & p-valor \\
\hline Variável & $r$ & $<0,001$ \\
\hline Classe NYHA & 0,590 & 0,003 \\
\hline Frequência cardíaca & 0,192 & $<0,001$ \\
\hline Tempo de hospitalização & 0,468 & $<0,001$ \\
\hline Escore de Mehran & 0,763 & $<0,001$ \\
\hline TFGe & $-0,671$ & \\
\hline
\end{tabular}

EuroSCORE II: European System for Cardiac Operative Risk Evaluation II; $r$ : coeficiente de correlação de log-rank de Spearman, NYHA: Classificação Funcional da New York Heart Association; TFGe: taxa de filtração glomerular estimada.

Tabela 3 - Achados do seguimento clínico em um ano

\begin{tabular}{lccc}
\hline Variável, $\mathbf{n}(\%)$ & CNP, $\mathbf{n = 2 5 ( 1 0 . 1 6 )}$ & CSFP, $\mathbf{n = 2 2 1 ( 8 9 . 8 4 )}$ & p-valor \\
\hline Mortalidade por todas as causas & $4(16)$ & $29(13,10)$ & 0,689 \\
\hline Morte Cardiovascular & $4(16)$ & $23(10,40)$ & 0,396 \\
\hline AVC & $6(24)$ & $6(2,70)$ & $<0,001$ \\
\hline Reinfarto do miocárdio & $3(12)$ & $25(11,30)$ & 0,918 \\
\hline ECAM & $11(44)$ & $51(23,10)$ & 0,022 \\
\hline
\end{tabular}

Os valores são expressos como números e porcentagens. CNP: fenômeno no-reflow coronariano; CSFP: fenômeno slow-flow coronariano; AVC: acidente vascular cerebral; ECAM: Eventos cardiovasculares adversos maiores.

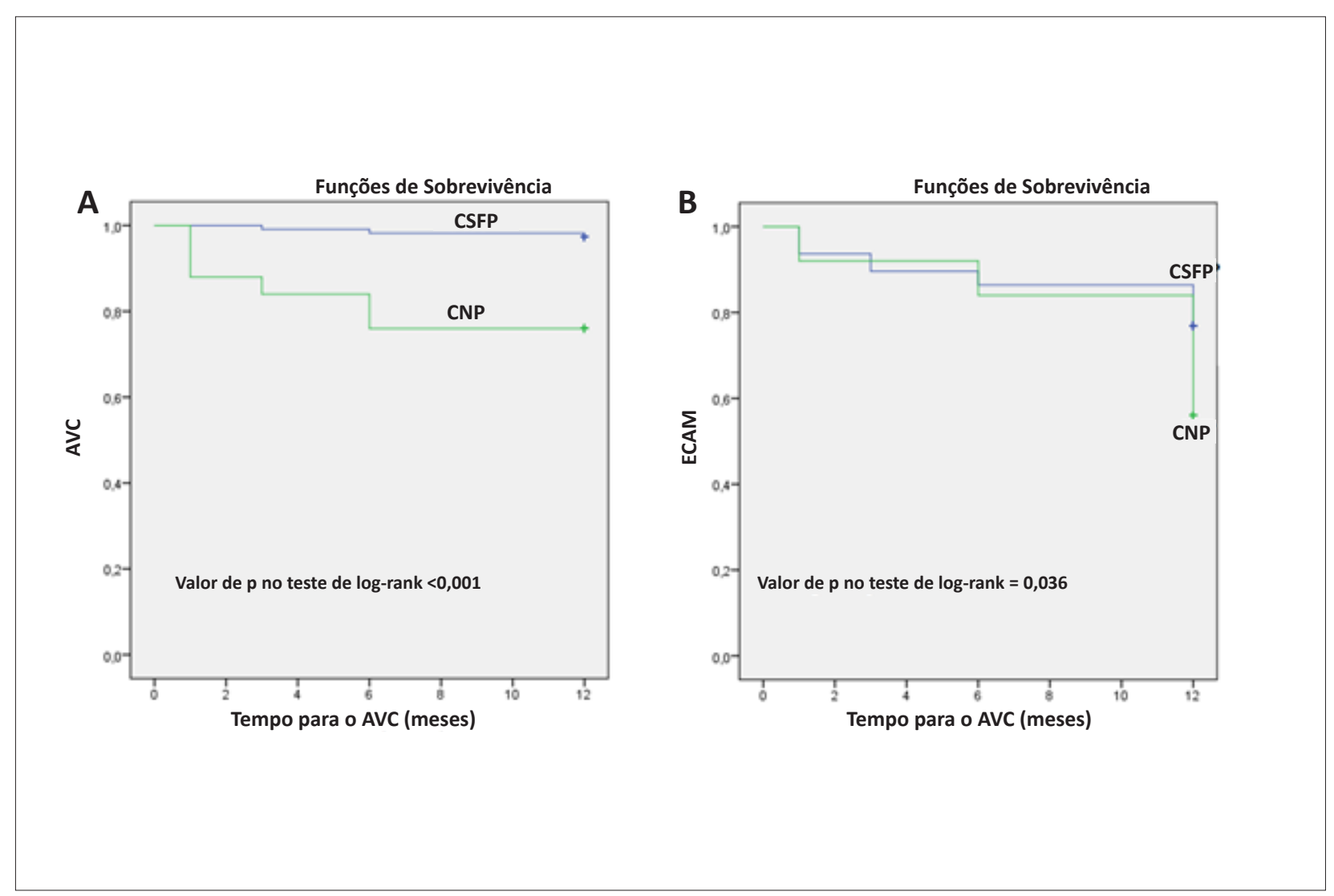

Figura 2 - (A) Estimativas de Kaplan-Meier para AVC. (B) Estimativas de Kaplan-Meier para ECAM. AVC: acidente vascular cerebral; ECAM: eventos cardiacos adversos maiores; CNP: fenômeno no-reflow coronariano; CSFP: fenômeno slow-flow coronariano. 


\begin{tabular}{lccc}
\hline Tabela 4 - Preditores independentes de CNP & & & \\
\hline Variável & OR & IC95\% & p-valor \\
\hline IMC & 1,11 & $1,00-1,24$ & 0,038 \\
\hline FC & 0,923 & $0,88-0,96$ & $<0,001$
\end{tabular}

OR: Odds ratio; IC: intervalo de confiança; IMC: indice de massa corporal; FC: frequência cardíaca.

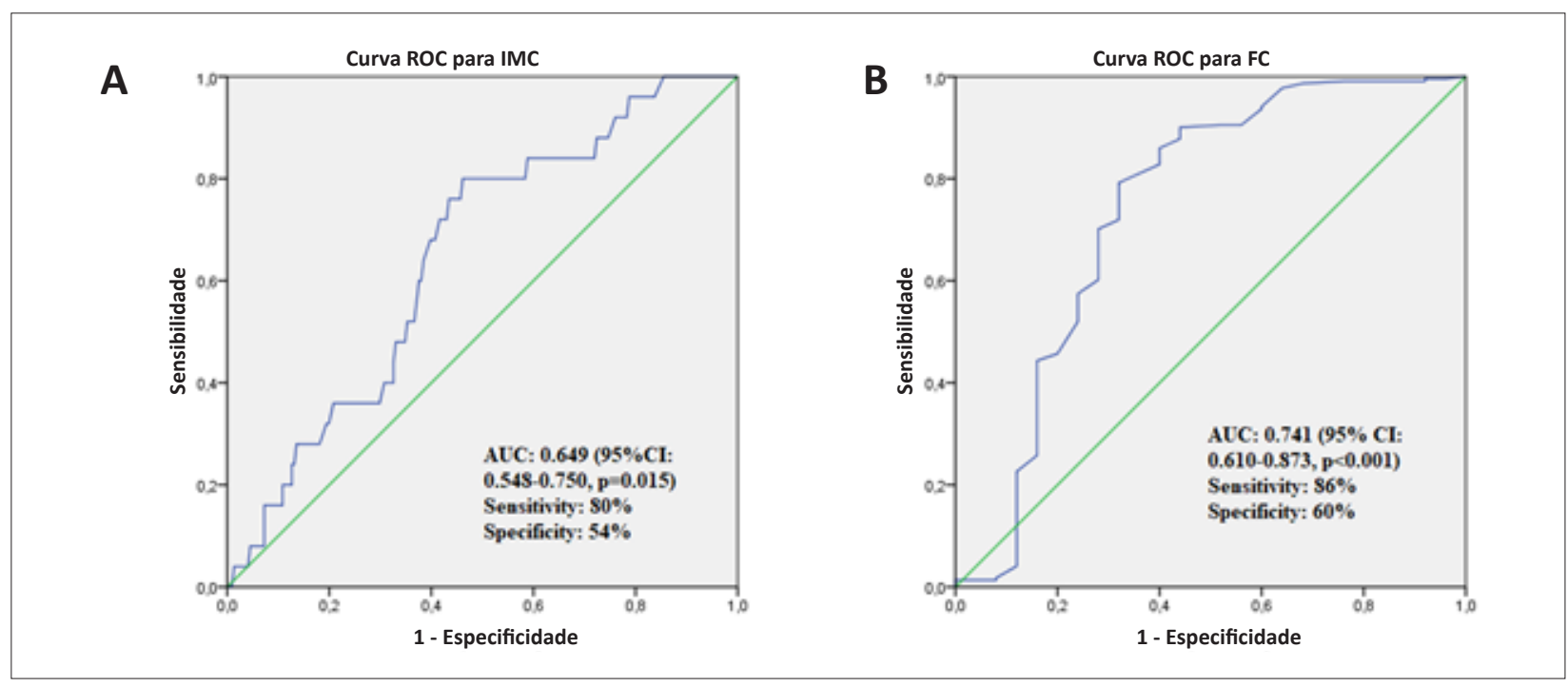

Figura 3 - (A) Curva ROC para a especificidade e sensibilidade do IMC. (B) Curva ROC para a especificidade e sensibilidade da FC. IMC: índice de massa corporal; FC: frequência cardíaca; ROC: Curva da característica de operação do receptor; AUC: área sob a curva; IC: intervalo de confiança.

AVC ocorreu com uma incidência significativamente mais alta no grupo CNP. Assim, após a alta, tais pacientes precisam ser cuidadosamente monitorados. Além disso, o IMC é o método mais comumente utilizado para avaliação de risco cardiovascular e obesidade. ${ }^{18}$

Em pacientes com IAMSSST, Bakirci et al. ${ }^{19}$ descobriram que a gordura epicárdica, que está aumentada em pacientes obesos, está associada ao fluxo coronariano prejudicado. ${ }^{19}$ Estudos recentes sugeriram que o CNP é mais comumente visto em combinação com hiperglicemia, hipercolesterolemia e insuficiência renal leve a moderada. ${ }^{20}$ No presente estudo, encontramos níveis de TFGe significativamente mais baixas e escores de Mehran mais altos no grupo CNP, consistentes com a literatura. Além disso, em nosso estudo, os pacientes do grupo CNP apresentaram IMC significativamente maior e consideramos que isso pode estar associado a um risco aumentado de acidente vascular cerebral. Portanto, o cálculo do IMC pode ser um método útil para estimar os desfechos cardíacos em pacientes com IAMSSST e CNP. Também consideramos que a diminuição do IMC pode proteger os pacientes contra um AVC.

Enquanto isso, estudos randomizados mostraram que o uso de cateter de aspiração manual de trombo pode fornecer melhor perfusão microvascular e resultados em longo prazo em comparação com pacientes controle. ${ }^{21}$ Entretanto, o uso de aspiração de trombo pode causar acidente vascular cerebral devido a complicações do dispositivo, razão pela qual em nosso estudo excluímos os pacientes $(n=6)$ submetidos a aspiração de trombo durante o procedimento índice, de forma que não afetasse o endpoint de AVC. O uso rotineiro de inibidores de plaquetas (inibidores da Gp-Ilb / IIla., Abciximab, tirofiban), nicorandil, nitroprussiato e adenosina mostraram efeitos benéficos na perfusão miocárdica no IAMSSST. ${ }^{22}$ Além disso, Aksu et al. descobriram que o uso de epinefrina intracoronária teve um efeito benéfico no $\mathrm{CNP}^{23}$ Além disso, Skelding et al. ${ }^{24}$ descobriram que um aumento da pressão arterial na circulação coronariana e taquicardia podem ser outros efeitos benéficos potenciais da epinefrina. ${ }^{24} \mathrm{Em}$ nosso estudo, consistente com a literatura, descobrimos que a FC mais baixa foi independentemente associada com o CNP em pacientes com IAMSSST. Se a microcirculação for lenta, o CNP ocorre, e sugerimos que a FC mais baixa poderia ser um indicador de CNP em pacientes com IAMSSST. Os médicos intervencionistas devem estar cientes da FC do paciente, e o paciente com menor FC deve ser considerado como candidato a CNP antes de iniciar a ICP. Apesar dos resultados encorajadores de nosso estudo, os achados de FC mais baixa devem ser explicados por estudos grandes e randomizados.

\section{Limitações}

Em primeiro lugar, embora um modelo multivariado tenha sido utilizado para ajustar as variáveis de confusão, um viés era inevitável, uma vez que sesta foi uma análise unicêntrica com um tamanho de amostra razoavelmente pequeno. Ensaios 
multicêntricos com mais pacientes podem mostrar melhores resultados e fornecer mais dados. Em segundo lugar, apenas parâmetros angiográficos foram usados na determinação do CNP e CSFP; a microcirculação não foi avaliada diretamente; por outro lado, nem a ecocardiografia, nem os pacientes foram avaliados com ressonância magnética (RM) para confirmar a reperfusão microvascular adequada. A RM é o melhor método para avaliação da obstrução microvascular. Terceiro, para avaliar os resultados clínicos em longo prazo, um período de seguimento de um ano pode não ser adequado. Esses fatores limitam nosso estudo.

\section{Conclusão}

Os dois determinantes do CNP em pacientes com IAMSSST foram níveis aumentados de IMC e FC mais baixa. Em nosso estudo, os resultados do seguimento clínico de um ano mostraram que a incidência de AVC e ECAM foi significativamente mais elevada no grupo CNP. Este estudo mostrou que o CNP piorou os desfechos dos pacientes com IAMSSST.

\section{Referências}

1. Fox KA, Cokkinos DV, Deckers J, Keil U, Maggioni A, Steg G. The ENACT study: a pan-European survey of acute coronary syndromes. European Network for Acute Coronary Treatment. Eur Heart J. 2000;21(17):1440-9.

2. Savonitto S, Ardissino D, Granger CB, Morando G, Prando MD, Mafrici A, et al. Prognostic value of the admission electrocardiogram in acute coronary syndromes. JAMA. 1999;281(8):707-13.

3. Jaffe R, Dick A, Strauss BH. Prevention and treatment of microvascular obstruction-related myocardial injury and coronary no-reflow following percutaneous coronary intervention: a systematic approach. JACC Cardiovasc Interv. 2010;3(7):695-704.

4. Brosh D, Assali AR, Mager A, Porter A, Hasdai D, Teplitsky I, et al. Effect of no-reflow during primary percutaneous coronary intervention for acute myocardial infarction on six-month mortality. Am J Cardiol. 2007;99(4):442-5

5. Hawkins BM, Stavrakis S, Rousan TA, Abu-Fadel M, Schechter E. Coronary slow flow-prevalence and clinical correlations. Circ J. 2012;76(4):936-42. Epub 2012 Feb 1.

6. Rezkalla SH, Stankowski RV, Hanna J, Kloner RA. Management of No-Reflow Phenomenon in the Catheterization Laboratory. JACC Cardiovasc Interv. 2017 Feb 13;10(3):215-23.

7. Thygesen K, Alpert JS, Jaffe AS, Chaitman BR, Bax JJ, Morrow DA, et al. Fourth universal definition of myocardial infarction (2018). Eur Heart J. 2019 Jan $14 ; 40(3): 237-69$.

8. Armstrong C, Joint National Committee. JNC 8 Guidelines for the Management of Hypertension in Adults, Am Fam Physician 2014 Oct $1 ; 90(7): 503-4$

9. Expert Committee on the Diagnosis and Classification of Diabetes Mellitus. Report of the expert committee on the diagnosis and classification of diabetes mellitus. Diabetes Care. 2003;26(Suppl 1): S5-20.

10. National Cholesterol Education Program Expert Panel on Detection Evaluation, and Treatment of High Blood Cholesterol in Adults. Third Report of the National Cholesterol Education Program expert panel on detection, evaluation, and treatment of high blood cholesterol in adults final report. Circulation. 2002;106(2):3143-21.

\section{Contribuição dos Autores}

Concepção e desenho da pesquisa; Obtenção de dados; Análise e interpretação dos dados; Análise estatística; Obtenção de financiamento; Redação do manuscrito; Revisão crítica do manuscrito quanto ao conteúdo intelectual importante: Huyut MA.

\section{Potencial Conflito de Interesses}

Declaro não haver conflito de interesses pertinentes.

\section{Fontes de Financiamento}

O presente estudo não teve fontes de financiamento externas.

\section{Vinculação Acadêmica}

Não há vinculação deste estudo a programas de pósgraduação.

11. Acquatella H, Asch FM, Barbosa MM, Barros M, Bern C, Cavalcante JL, et al. Recommendations for Multimodality Cardiac Imaging in Patients with Chagas Disease. J Am Soc Echocardiogr. 2018 Jan;31(1):3-25.

12. Gibson CM, Cannon CP, Daley WL, Dodge JT Jr, Alexander B Jr, Marble SJ, et al. TIMI frame count: a quantitative method of assessing coronary artery flow. Circulation 1996 Mar 1;93(5):879-88.

13. Durante A, Camici PG. Novel insights into an "old" phenomenon: the no reflow. Int J Cardiol. 2015;187:273-80.

14. Chaudhry MA, Smith M, Hanna EB, Lazzara R. Diverse spectrum of presentation of coronary slow flow phenomenon: a concise review of the literature. Cardiol Res Pract. 2012;2012:383181.

15. Jaffe R, Dick A, Strauss BH. Prevention and treatment of microvascular obstruction-related myocardial injury and coronary no-reflow following percutaneous coronary intervention: a systematic approach. JACC Cardiovasc Interv. 2010;3(7):695-704.

16. Ndrepepa G, Tiroch K, Fusaro M, Keta D, Seyfarth M, Byrne RA, et al. 5-year prognostic value of no-reflow phenomenon after percutaneous coronary intervention in patients with acute myocardial infarction. J Am Coll Cardiol. 2010 May 25;55(21):2383-9.

17. Nienhuis MB, Ottervanger JP, Bilo HJ, Dikkeschei BD, Zijlstra F. Prognostic value of troponin after elective percutaneous coronary intervention: A metaanalysis. Catheter Cardiovasc Interv 2008;71(3):318-24.

18. Bergman RN, Kim SP, Catalano KJ, Hsu IR, Chiu JD, Kabir M, et al. Why visceral fat is bad: mechanisms of the metabolic syndrome. Obesity (Silver Spring). 2006 Feb;14(suppl 1):16-9.

19. Bakirci EM, Degirmenci H, Duman H, Inci S, Hamur H, Buyuklu M, et al. Increased epicardial adipose tissue thickness is associated with angiographic thrombus burden in the patients with non-st-segment elevation myocardial infarction. Clin Appl Thromb Hemost. 2015 Oct;21(7):612-8.

20. Ipek G, Onuk T, Karatas MB, Gungor B, Osken A, Keskin M, et al. CHA2DS2VASc score is a predictor of no-reflow in patients with ST-segment elevation myocardial infarction who underwent primary percutaneous intervention. Angiology. 2016 Oct; 67(9):840-5 
21. Vlaar PJ, Svilaas T, van der Horst IC, Diercks GF, Fokkema ML, de Smet $\mathrm{BJ}$, et al. Cardiac death and reinfarction after 1 year in the thrombus aspiration during percutaneous coronary intervention in acute myocardial infarction Study (TAPAS): a 1-year follow-up study. Lancet. 2008 Jun 7;371(9628):1915-20.

22. Amit G, Cafri C, Yaroslavtsev S, Fuchs S, Paltiel O, Abu-Ful A, et al. Intracoronary nitroprusside for the prevention of the no-reflow phenomenon after primary percutaneous coronary intervention in acute myocardial infarction. Am Heart J. 2006 Nov;152(5):887. e9-14.
23. Aksu T, Guler TE, Colak A, Baysal E, Durukan M, Sen T, et al. Intracoronary epinephrine in the treatment of refractory no-reflow after primary percutaneous coronary intervention: a retrospective study. BMC CardiovasC Disord. 2015 Feb 19;15:10.

24. Skelding KA, Goldstein JA, Mehta L, Pica MC, O'Neill WW. Resolution of refractory no-reflow with intracoronary epinephrine. Catheter Cardiovasc Interv. 2002 Nov; 57(3):305-9. 